\title{
EL TRABAJO RÍTMICO REALIZADO A TRAVÉS DE LA MÚSICA: UNA HERRAMIENTA PARA LA REHABILITACIÓN DE NIÑOS/AS CON DISLALIA FUNCIONAL
}

\author{
$M^{a}$ Esther Ruiz Palomo ${ }^{1}$ \\ Facultad de Humanidades y Educación \\ Universidad de Burgos \\ Fernando Lara Ortega ${ }^{2}$ \\ Facultad de Humanidades y Educación \\ Universidad de Burgos
}

Recibido: 15/3/2014

Aceptado: 27/4/2015

\section{Resumen:}

En este trabajo se muestran los resultados obtenidos en dos variables: Adaptación a un ritmo externo y repetición de estructuras rítmicas, tras aplicar, como intervención indirecta, un programa de música específicamente diseñado para mejorar las bases funcionales del lenguaje oral, a un grupo experimental de sujetos con dislalia funcional. Dichos resultados se han extraído de una investigación más amplia, realizada según un diseño pre-test-rehabilitación-post-test, en la que durante cinco meses el grupo experimental recibió clases de música una vez por semana, además de la sesión de intervención directa con la logopeda. El grupo control no recibió clases de música y continuó con su tratamiento logopédico habitual. La investigación incidía en 7 variables de las que se han seleccionado las ya mencionadas por su relevancia en los problemas tratados. Los resultados sugieren que el trabajo rítmico realizado a través de la música ha contribuido a rehabilitar las dislalias funcionales que presentaban.

Palabras clave: Reeducación, educación musical, logopedia, educación infantil, programa.

\begin{abstract}
:
In this work, it is shown the results obtained within two variables: Adaptation to an external rhythm and repetition of rhythmic structures, after implementing, as indirect intervention, a music programme specially designed to improve the functional basis of oral language, to an experimental group of subjects with functional difficulties of articulating the words. Those results have been extracted from a comprehensive research made according to a pre-test- rehabilitation- post-test design, in which for five months the experimental group received music lessons once a week, in addition to the direct intervention session with speech therapist. The control group doesn't received music lessons and continued his usual speech therapy. The research touched on 7 variables; from those variables, we have selected the two mentioned above because they have a specific relevance on the dysfunctions treated. The results suggest that the rhythmic work through music has helped rehabilitate the difficulties presenting.
\end{abstract}

Keywords: Re-education, musical education, speech therapy, child education, programme.

\footnotetext{
1 erpalomo@ubu.es

2 flara@ubu.es
} 
Un considerable número de investigaciones ponen de manifiesto los beneficios de la educación musical en el desarrollo lingüístico. Todas ellas señalan que la música puede facilitar el lenguaje expresivo y ayudar al desarrollo del lenguaje receptivo.

Los resultados obtenidos en la investigación realizada por Herrera, HernándezCandelas, Lorenzo y Ropp ponen de manifiesto: “que el entrenamiento musical puede marcar una diferencia crítica en el desarrollo general infantil, especialmente en términos de lenguaje.” (2014, p.13).

En uno de sus trabajos Ramírez Hurtado señala que: “si la música puede aportar algo importante en la intervención sobre el desarrollo del lenguaje en el aula ordinaria, parece lógico pensar que también lo hará cuando nos encontramos con dificultades, retrasos o problemas diversos en la adquisición y desarrollo del lenguaje.” (2006, p. 255)

Dentro del campo de las necesidades específicas del lenguaje, los errores que con mayor frecuencia nos encontramos en los alumnos de Educación Infantil y primer ciclo de Primaria, son aquellos relacionados con la articulación, conocidos como dislalia funcional.

Aunque encontramos muchas definiciones de dislalia funcional podríamos decir que: "Las dislalias son alteraciones en la articulación de los fonemas, ocasionadas por una manifiesta incapacidad para pronunciar de forma adecuada determinados fonemas o grupos de fonemas, sin que se adviertan lesiones o malformaciones en los órganos periféricos del habla.” (Gallego, 2000, p. 15).

Si prestamos atención a las causas que pueden provocar la aparición de dislalias, aunque los distintos autores coinciden en admitir la concurrencia de varias como factores desencadenantes, son muchos (Busto Barcos, 1998; García-Valdecasas, 2001; Juárez y Monfort, 1995; Martínez Agudo, 2002; Veiga Liz, 2004)) los que señalan entre ellas dificultades psicomotrices y una escasa habilidad motora.

Igualmente la evaluación que se realiza previa a la intervención incluye, entre otros aspectos, una evaluación de la motricidad y del tono muscular, y desde la intervención también se concede importancia al trabajo rítmico.

El Modelo fonético de intervención utiliza dos estrategias basándose en las características de cada fonema: 
1. Intervención directa, en la cual el logopeda interviene intentando la producción correcta del fonema.

2. Intervención indirecta, donde las bases funcionales de la articulación son intervenidas antes de pasar a la intervención directa. Las actividades a llevar a cabo dentro de esta forma de intervención incluyen, entre otras, las referidas a motricidad: motricidad general, ritmo y motricidad bucofacial.

El ritmo es un elemento esencial de la música e, igualmente, una cualidad del lenguaje hablado, por lo que adquiere gran importancia en el tratamiento de dislalias ya que ayuda a fijar y automatizar los esquemas corporales de la correcta articulación (Barrena González, 2006). El ritmo métrico, basado en la sucesión de pulsos y acentos, es imprescindible para las ordenaciones temporales y la coordinación de movimientos, ayudando a desarrollar el control motriz y la coordinación. Todo ello puede conseguirse trabajando el ritmo a través del movimiento corporal y del manejo de sencillos instrumentos musicales.

Unido a ello las secuencias rítmicas que se originan en retahílas, canciones, etc., y el trabajo prosódico, permitirán conectar con la habilidad rítmica necesaria para la emisión de un mensaje oral y trabajar las habilidades del habla fuera del contexto verbal pero respetando sus principales características: acentuación, pausa, etc. (Juárez y Monfort, 2001).

El trabajo a través de un programa de música debidamente diseñado y aplicado en forma de intervención indirecta, aporta una nueva dimensión, pues favorece el desarrollo de la expresión no verbal, del área socio-afectiva y del área perceptivocognitiva (Ramírez Hurtado, 2006), a la vez que permite trabajar muchos de los aspectos señalados por Guberina (http://www.verbotonal.org/sistemaverbotonal/metodo-verbotonal.html) como parámetros del habla tales como: entonación, intensidad, pausa, tempo y ritmo de habla, elementos que constituyen también parámetros del sonido y componentes fundamentales del discurso musical.

Esta aplicación de la música se hace posible al incorporar los principios de la Musicoterapia - que ayudan a valorar las realizaciones musicales desde una perspectiva 
más abierta- a los métodos de educación musical, más enfocados al desarrollo de competencias musicales específicas (Sabbatella, 2001), es decir, se trata de emplear la música como un medio para lograr objetivos reeducativos y no como un fin, ya que en este caso lo importante no es el aprendizaje de contenidos ni el desarrollo de destrezas musicales.

Para enmarcar la intervención dentro de los supuestos naturalistas o ecológicos (Acosta y Moreno, 2003; Castejón y España, 2004; Vilaseca, 2002):

a. Trabajamos con niños de edades comprendidas entre 4-6 años, dada la importancia que se concede a la intervención temprana en la literatura científica.

b. Realizamos una intervención en grupo para situar la investigación en un contexto más próximo a situaciones reales de comunicación y porque el tratamiento en grupo estimula el lenguaje, desarrolla habilidades para la comunicación, fomenta el diálogo, etc., (Galán, 2001)

c. Llevamos a cabo la intervención en el contexto escolar: 1. por la importancia adquirida por la logopedia escolar, 2. porque el Centro Escolar es considerado por la mayoría de los profesionales como el marco ideal para la detección e intervención temprana de las dificultades del lenguaje, y 3. porque permite que todos los niños participantes estén influidos por las mismas experiencias además de favorecer la homogeneidad de los grupos.

El presente trabajo se realizó en un Centro de Educación Infantil y Primaria de Burgos partiendo de la hipótesis de que los niños con problemas funcionales de habla que, además de recibir intervención logopédica directa son intervenidos de forma indirecta mediante un programa de música específicamente diseñado, mejorarán respecto al grupo de control en una serie de variables: habilidad buco-linguo-labial, respiración, suficiencia nasal, soplo, discriminación auditiva, y habilidades rítmicas que incluye dos variables: adaptación a un ritmo externo y repetición de estructuras rítmicas, todas ellas compuestas a su vez por varios ítems.

El objetivo fundamental del trabajo es incidir, a través de la música, en las bases funcionales que causan problemas de habla, para lo cual diseñamos un programa de 
quince sesiones enmarcado dentro de la intervención indirecta en el tratamiento de los problemas de habla.

De este objetivo fundamental se derivan dos objetivos más específicos:

- Elaborar un programa de intervención musical que posibilite la mejora de: habilidades rítmicas (adecuación a un ritmo externo, tempo espontáneo y repetición de estructuras rítmicas), habilidad buco-linguo-labial (articulación), respiración, suficiencia nasal, soplo y discriminación auditiva en niños con dislalia funcional.

- Evaluar dicho programa.

Entre las variables trabajadas hemos seleccionado aquellas relacionadas con el ritmo: Adaptación a un ritmo externo y Repetición de estructuras rítmicas, por su destacada importancia en el problema tratado: dislalia funcional, ya que, como señala Barrena González (2006, parte VI, p. 1): “Por la estrecha relación que existe entre el movimiento y el habla, se detecta en muchos casos cómo un retraso motor puede ser un factor etiológico que desencadena o favorece la aparición de un dislalia funcional.”

\section{MÉTODO}

\section{Participantes}

Se trabajó con niños de un Centro Escolar Público de Burgos todos ellos con problemas de habla diagnosticados por los equipos psicopedagógicos y por los logopedas del propio Centro Escolar.

Para la selección de la muestra (tanto del grupo control como del grupo experimental) se recurrió a los logopedas, ya que los sujetos debían, obviamente, presentar dislalias funcionales diagnosticadas y recibir atención logopédica en el propio Centro Escolar.

También se tuvo en cuenta:

o la edad de los sujetos, que debía estar entre 4 y 7 años y ser lo más uniforme, 
o el número de sujetos de ambos grupos, que consideramos debería ser de aproximadamente ocho alumnos.

La necesidad de adaptarnos a la planificación de la logopeda supuso una modificación del proyecto inicial de tal manera que, en lugar de contar con un único grupo experimental, tuvimos que fragmentar la muestra en dos grupos: uno compuesto por cinco niños de 5 años y otro con cuatro niños de 6 años (Ver Tabla 1)

Esto nos obligó a buscar grupos de control de las mismas características y, aunque no logramos que tuvieran el mismo número de niños, al menos sí pudimos conseguir que tuvieran edades similares.

Finalmente la muestra quedó distribuida como sigue:

\begin{tabular}{|l|l|l|}
\hline & 2 Grupos experimentales & 2 Grupos control \\
\hline Composición & 4 (5 años) / 5 (6 años) & 3 (5 años) / 4 (6 años) \\
\hline
\end{tabular}

Tabla 1: Distribución de la muestra

Nuestro ámbito de actuación fue $3^{\circ}$ de Educación Infantil y $1^{\circ}$ de Educación Primaria. En el Centro Escolar en el que se ha desarrollado la investigación eran atendidos en aquel momento 34 niños de ambos cursos por necesidades específicas del lenguaje no asociadas a otra discapacidad. En nuestra investigación trabajamos con 26, es decir, más de un 76\% de los niños que reciben atención logopédica por dislalias, por lo que creemos que la muestra, pese a ser pequeña, sí es representativa.

\section{Instrumentos}

\section{Instrumento de medida}

Para recopilar datos, tanto en el pre-test como en el post-test aplicamos un instrumento de medida (Apéndice A) elaborado con extractos de tres pruebas estandarizadas para la evaluación de dislalias funcionales: ELCE (Exploración del lenguaje comprensivo y expresivo), PAF (Prueba de Articulación de fonemas) y Prueba para la exploración diagnóstica del desarrollo del lenguaje oral. 
Concretamente las subpruebas para la evaluación de las variables que nos ocupan: Adecuación a un ritmo externo: lento-normal-rápido y Repetición de estructuras rítmicas, fueron extraídas de la prueba ELCE (Exploración del lenguaje comprensivo y expresivo) (López Gines, 1996).

1. En Adecuación a un ritmo externo: lento-normal-rápido, se valora la capacidad del sujeto para adaptar su movimiento a un ritmo externo producido por el evaluador mediante un pandero y la facilidad de transición de uno a otro.

2. En Repetición de estructuras rítmicas Se trata de medir la capacidad del sujeto para repetir correctamente una serie de siete estructuras rítmicas, ya establecidas, propuestas por el examinador.

\section{Guía de observación y tabla de valoración}

Complementamos la recopilación de datos con otros métodos tales como: observación participante, por medio de Guías de observación elaboradas por la investigadora (Apéndice B), entrevistas con las tutoras de los niños y valoraciones de la logopeda (Apéndice C).

\section{Programa de intervención}

Para la rehabilitación se aplicó un programa, diseñado y llevado a cabo por la investigadora. Dicho programa se estructuraba en quince sesiones que alternaban con las sesiones habituales de tratamiento directo. En ellas se trabajaban aspectos tales como: respiración, articulación, vocalización, entonación, discriminación auditiva, formación rítmica, etc., es decir, aquellos que constituyen las bases funcionales del habla y que entroncan con contenidos de educación musical.

Paralelamente las canciones seleccionadas servían para reforzar la intervención directa al trabajar, en la medida de lo posible, los mismos fonemas que lo logopeda trabajaría en la sesión. 
Los resultados de las pruebas del pre-test marcaron las líneas en las que debíamos hacer más hincapié, y que pasaron a ser los contenidos del programa, que, en líneas generales están en relación con:

- Ritmo y habilidades motrices: adaptación del movimiento a una música o a un estímulo sonoro externo, sencillas danzas, prosodia de la letra de las canciones, repetición por imitación de pequeños esquemas rítmicos con percusión corporal, etc.

- Percepción y discriminación de estímulos auditivos: escuchar y reconocer sonidos del entorno, clasificar/relacionar sonidos según sus cualidades, etc.

- Técnica vocal: distintos ejercicios de articulación y vocalización, respiración, soplo y emisión, relajación y entonación seleccionados atendiendo al fonema que se trabaje en la sesión

- Canciones. Canciones populares infantiles españolas o sencillas canciones creadas por la investigadora según las necesidades de rehabilitación, seleccionadas en función del fonema a trabajar y con unas características musicales tales que facilitasen su aprendizaje y realización por parte de los niños, habida cuenta de que nuestro objetivo no era la excelencia en la interpretación de las mismas.

En el trabajo de los contenidos no sólo se empleó la música, sino que también empleamos el sonido en su vertiente más variada de actividades relacionadas con la producción sonora, la discriminación, asociación, juegos sonoros, representaciones gráficas y corporales, etc.

Las sesiones tenían una duración de 50’y se planificaron tomando como base el proceso metodológico recomendado por Gallardo y Gallego (2003):

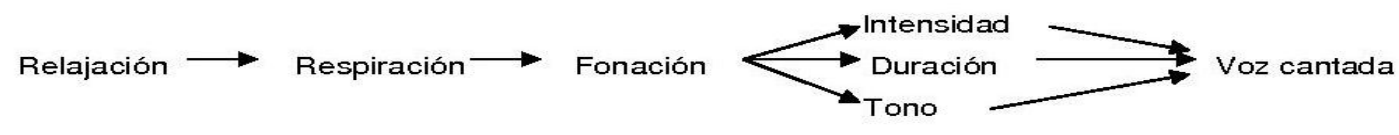

Partiendo de él las sesiones se organizaron como sigue:

Relajación - Respiración - Educación vocal - Canción - Juegos de percepción auditiva - Actividades rítmicas. Todas las actividades se diseñaban en función de la canción, que variaba en cada sesión para reforzar la intervención directa en los diferentes fonemas acordados previamente con la logopeda, de acuerdo con los problemas que presentaban los sujetos. 


\section{Análisis de datos}

Para el análisis de datos utilizamos una combinación de técnicas cualitativas y cuantitativas.

En el análisis cualitativo fuimos seleccionando y abstrayendo datos, para ir estableciendo conclusiones tras cada sesión y de cada uno de los sujetos del grupo experimental, mediante Guías de observación elaboradas por la investigadora (Apéndice B). Así mismo, la logopeda realizó una valoración final (Apéndice C) de cada uno de los niños del grupo experimental que nos ha ayudado en el análisis cualitativo de los datos.

Para el análisis cuantitativo aplicamos tratamientos estadísticos a los datos obtenidos, empleando la Prueba de los rangos con signo de Wilcoxon del programa SPSS

\section{Procedimiento}

El procedimiento de intervención se basó en un diseño clásico: pre-testrehabilitación-post-test. El tiempo transcurrido entre pre-test y post-test, empleado para aplicar el programa, fue de cinco meses.

El grupo experimental recibía dos sesiones semanales, una sesión grupal en la que trabajaba con el programa y una sesión individual con la logopeda, mientras que el grupo de control trabajaba únicamente con la logopeda, realizando las dos sesiones individuales habituales, sin participar en el programa.

Las pautas de actuación vinieron dadas por la necesidad de adaptarnos al contexto en el que se iba a llevar a cabo el trabajo; no obstante para guiar el estudio, lo dividimos en etapas, como sigue:

- Elección del Centro Escolar en el que queríamos llevar a cabo la experiencia y entrevistas con los responsables del mismo.

- Selección de la muestra, de acuerdo con la logopeda del Centro Escolar, y valoración inicial (pre-test) del grupo experimental y del grupo de control. Diseño del programa de acuerdo con los resultados obtenidos. 
- Aplicación del programa de música con el grupo experimental, con un total de 15 sesiones.

- Post-test, análisis de datos y evaluación del programa.

- Conclusiones.

\section{RESULTADOS}

Los resultados obtenidos tras la aplicación del programa muestran mejoras en la mayoría de los ítems medidos, lo que nos hace suponer la eficacia del mismo aplicado como intervención indirecta en el tratamiento logopédico de las dislalias funcionales.

Para el estudio objetivo de dichos resultados llevamos a cabo dos tipos de análisis: análisis cuantitativo y análisis cualitativo.

\section{Análisis cuantitativo}

Consistió en la comparación de los resultados obtenidos en el pre-test con los del post-test, tras aplicar el programa, empleando el mismo instrumento de medida.

En relación con el grupo de control, que no realizó el programa, no se apreciaron cambios significativos. En cuanto al grupo experimental el tratamiento contribuyó a que se apreciaran diferencias estadísticamente significativas en varios de los ítems.

\section{Adecuación a un ritmo externo}

Se evaluaban tres ítems: “adecuación a un ritmo lento”, “adecuación a un ritmo normal” y “adecuación a un ritmo rápido”.

Al estudiar los resultados de las pruebas de pre-test, vimos que en la prueba "adecuación a un ritmo lento" fallaban cinco niños del grupo de control y siete del grupo experimental. En el post-test dos de los siete sujetos del grupo experimental que fallaron inicialmente en la prueba la llevaron a cabo con éxito.

La prueba de “adecuación a un ritmo rápido”, fue llevada a cabo correctamente por todos los niños del grupo experimental, aunque encontramos tres fallos en el grupo de control, situación que no varió en el post-test, y la prueba de “adecuación a un ritmo 
normal" sólo contó en el pre-test con un fallo en cada uno de los grupos aunque en el post-test el sujeto del grupo experimental que falló la realizó correctamente.

En ninguno de los casos los resultados muestran diferencias significativas entre pre-test y post-test.

\section{Repetición de estructuras rítmicas}

Las diferencias iniciales que se observaban entre ambos grupos no eran muy destacables. El nivel en la realización de las pruebas en el pre-test fue muy similar en los dos, no habiendo ningún niño capaz de repetir correctamente la totalidad de dichas estructuras.

En el post-test sin embargo encontramos en el grupo experimental un incremento importante en el número de sujetos que han respondido de forma adecuada.

Así podemos señalar que:

- La mejora menos importante, un sujeto, se produce en la repetición de las estructuras “estr1: ...”, y “estr5: ....”.

- La segunda estructura, “estr2: .. ...”, (Tabla 2) fue realizada correctamente en el pretest por tres niños del grupo experimental, y en el post-test todos lograron superarla, lo que da como resultado una diferencia pre-test- post-test muy significativa, con un valor para el estadístico de contraste de .014.

\section{Rangos}

\begin{tabular}{|c|c|c|c|c|}
\hline \multicolumn{2}{|l|}{ Grupo (experimental/control) } & $\mathrm{N}$ & $\begin{array}{c}\text { Rango } \\
\text { promedio }\end{array}$ & Suma de rangos \\
\hline \multirow[t]{4}{*}{ Experimental } & Rangos negativos & $0^{\mathrm{a}}$ & .00 & .00 \\
\hline & Rangos positivos & $6^{\mathrm{b}}$ & 3.50 & 21.00 \\
\hline & Empates & $3^{c}$ & & \\
\hline & Total & 9 & & \\
\hline \multirow[t]{4}{*}{ Control } & Rangos negativos & $0^{\mathrm{a}}$ & .00 & .00 \\
\hline & Rangos positivos & $0^{\mathrm{b}}$ & .00 & .00 \\
\hline & Empates & $7^{c}$ & & \\
\hline & Total & 7 & & \\
\hline
\end{tabular}




\section{Rangos}

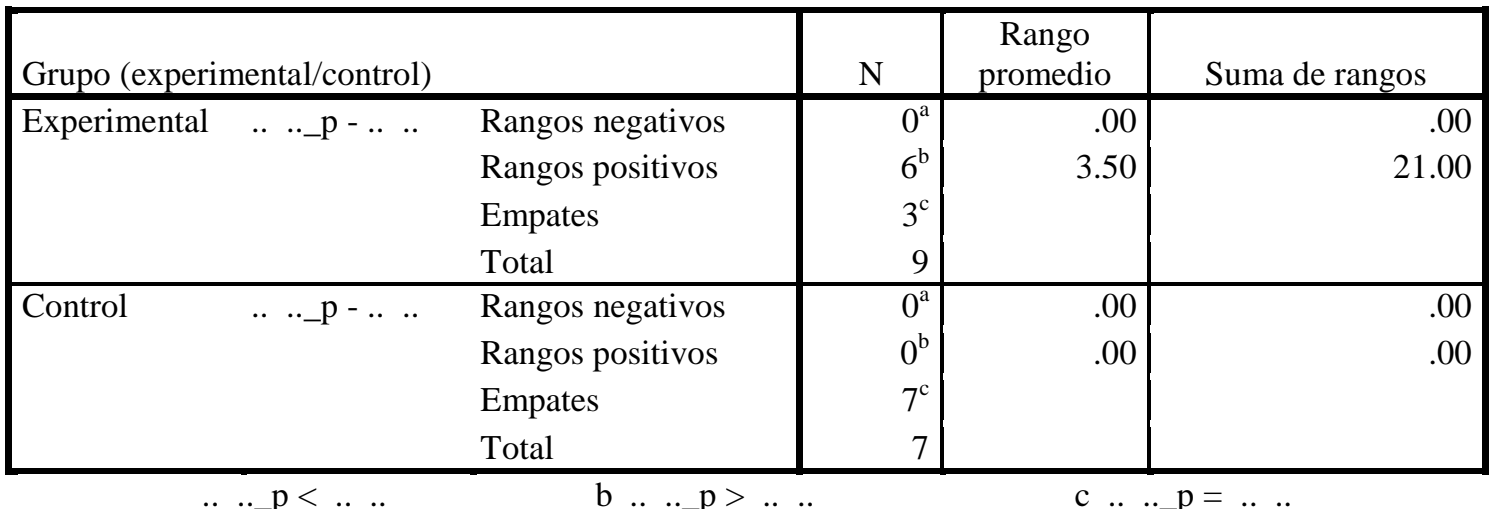

\begin{tabular}{|l|l|r|}
\hline \multicolumn{2}{|c|}{ Estadísticos de contraste $^{\mathrm{c}}$} \\
\hline \multirow{2}{*}{ Grupo (experimental/control) } & $\ldots$.. ..p - .. .. \\
\hline Experimental & Z & $-2.449^{\mathrm{a}}$ \\
\cline { 2 - 3 } & Sig. asintót. (bilateral) & .014 \\
\hline Control & Z & $.000^{\mathrm{b}}$ \\
\cline { 2 - 3 } & Sig. asintót. (bilateral) & 1.000 \\
\hline
\end{tabular}

c. Prueba de los rangos con signo de Wilcoxon

Tabla 2: Comparativa entre el G. E. y el G. C. en el ítem “Estr2: .. ..”.

1. Otro caso en el que encontramos una diferencia significativa (estadístico de contraste $=.025)$ se produce en la estructura cuatro, "estr4: . . .”, (Tabla 3) donde observamos que los cinco niños del grupo experimental que no pudieron llevarla a cabo adecuadamente en el pre-test, sí lo han hecho en el post-test.

\section{Rangos}

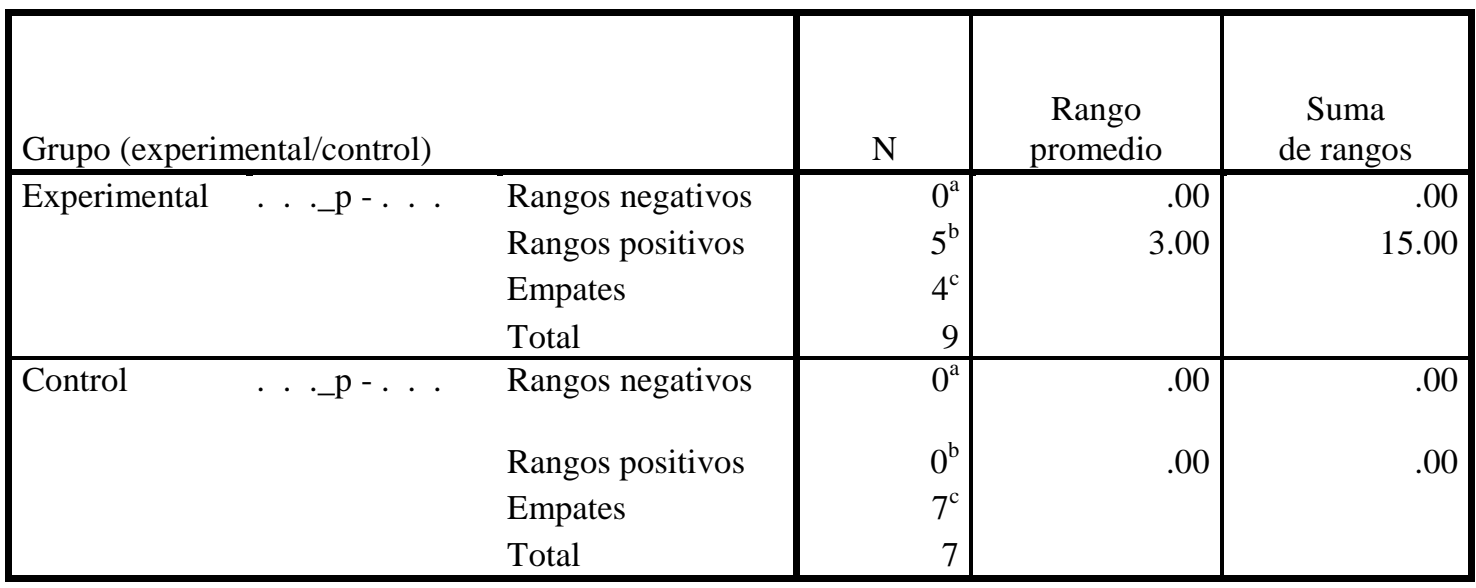




\section{Rangos}

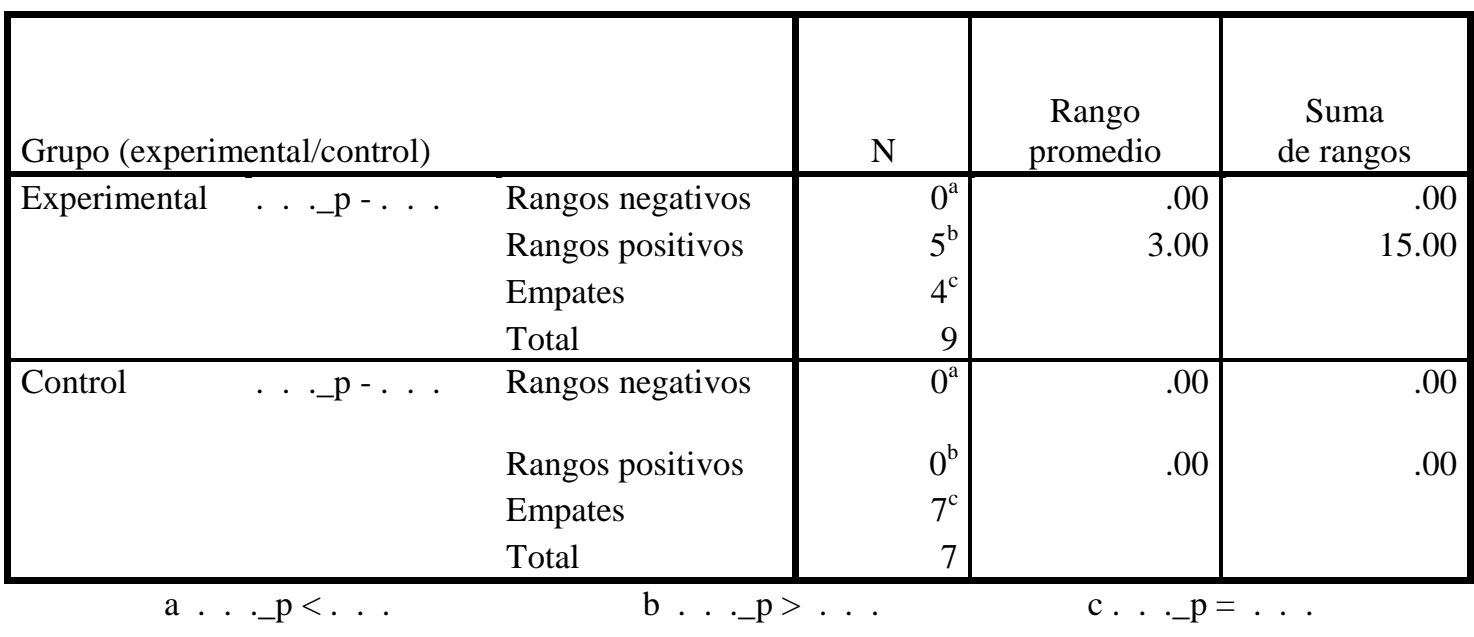

Estadísticos de contraste ${ }^{c}$

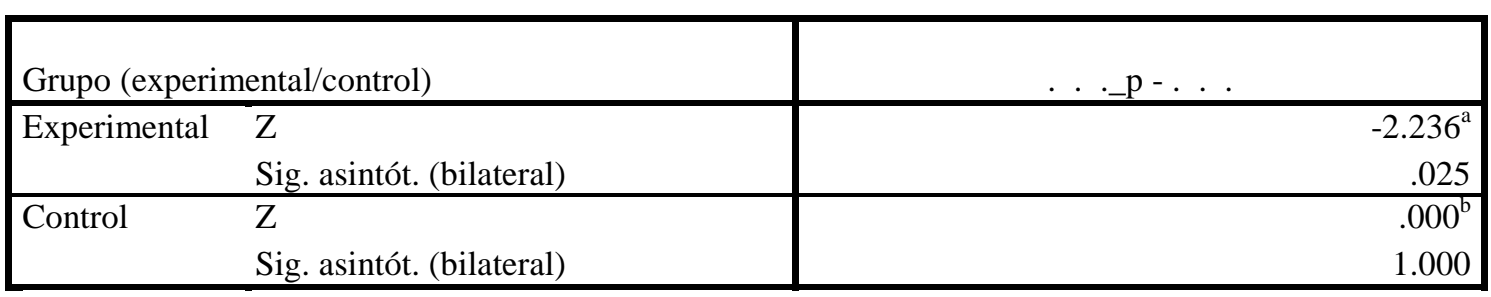

1. También son cinco los niños del grupo experimental que mejoran su realización de la prueba en la repetición de la estructura “estr7: .. . . ..” , y, aunque sigue habiendo dos que no lo han logrado, el valor del estadístico (.025) muestra también que la diferencia pre-test-post-test es significativa.

1) Por último, en las estructuras “estr3: . ..” y “estr6: . ...” cuatro niños del G.E. han pasado de incorrecto a correcto, quedando un niño y dos respectivamente sin haber resuelto la prueba de forma satisfactoria, a pesar de lo cual el valor del estadístico (.046) también indica que la diferencia con relación al pre-test es significativa.

\section{Análisis cualitativo}

De los resultados del análisis cualitativo también se desprende una valoración bastante positiva. Estudiando los datos obtenidos en la valoración final llevada a cabo por la logopeda (Tabla 4), podemos apreciar que todos los niños han experimentado una 
mejora, en mayor o menor grado, en los aspectos evaluados en relación con las variables aquí presentadas.

\begin{tabular}{|l|r|r|r|r|r|}
\hline & NADA & POCO & REGULAR & MUCHO & NOTABLEMENTE \\
\hline Ritmo y acentuación del habla & & 1 & 7 & 1 & \\
\hline Coordinación dinámica general & & & 8 & 1 & \\
\hline Articulación del/los fonema/s afectado/s & & 1 & & & \\
\hline
\end{tabular}

Tabla 4: Valoración realizada por la logopeda

Así mismo a través de las guías de observación se pudo ver que la tendencia general era la de una mejora progresiva en los aspectos trabajados en relación con las variables señaladas.

\section{DISCUSIÓN}

Los resultados indican que el trabajo rítmico a través de la música puede mejorar las bases funcionales del lenguaje oral y por tanto contribuir a la rehabilitación de dislalias funcionales.

En el análisis cuantitativo hemos podido apreciar que en todos los aspectos evaluados se han producido mejoras, y aquellos en los que no se han observado han sido los que inicialmente habían sido realizados correctamente por todos los sujetos del grupo experimental.

En la variable Repetición de estructuras rítmicas, dichas mejoras han sido significativas (mejoras de cuatro sujetos o más) en los ítems: “estr2: .. ..”, “estr3: ...”, “estr4: . ..”, “estr6: . ...” y “estr7: .. .. ...”.

Tal y como vimos, en el pre-test, excepto en dos de los ítems evaluados, se producen muchos errores en ambos grupos, sin embargo en el post-test comprobamos que los niños del grupo experimental mejoran prácticamente todos y en todos los ítems, mientras que los niños del grupo de control permanecen igual. 
El estudio de los valores estadísticos muestra que dichas diferencias significativas conllevan evoluciones positivas de entre cuatro y seis sujetos, en los cinco ítems anteriormente señalados.

Los valores estadísticos no significativos los encontramos en la repetición de las estructuras “estr1: ...” y “estr5: ....”, con sólo una mejora en cada una, pero en el pretest solamente se había producido un error en ellas, por lo que finalmente todos los niños realizaron la repetición de forma adecuada.

En cuanto a la variable Adecuación a un ritmo externo también, y como ya vimos, se han observado mejoras, aunque en ningún caso las diferencias con el pre-test son significativas, y creemos que no pueden atribuirse a la aplicación del programa, aunque sí es posible que las actividades desarrolladas en el mismo hayan estimulado el proceso madurativo, colaborando de forma importante a dichas mejoras.

La prueba en la que más errores se produjeron, cinco niños del grupo de control y siete del grupo experimental, fue "adecuación a un ritmo lento" posiblemente debido a que el “tempo” natural del niño es más rápido que el del adulto (aproximado a la marca metronómica de 100) por lo que adecuar su movimiento a un ritmo por debajo de esa marca supone para ellos una gran dificultad. A la vista de los resultados podemos inferir que el Programa no ha cubierto suficientemente este aspecto.

En relación con el análisis cualitativo también se pueden extraer conclusiones positivas dado que, tanto a través de la valoración de la logopeda como de las guías de observación se aprecia mejora en las variables estudiadas.

En las valoraciones llevadas a cabo por la logopeda al finalizar la intervención (Tabla 4), se observa que, siempre de acuerdo con su apreciación personal, 7 niños mejoran regular, 1 mucho y 1 que mejora poco, en ritmo y acentuación del habla, y 8 niños mejoran regular y uno mucho en coordinación dinámica general.

Puesto que la logopeda no poseía conocimientos musicales, por lo cual le resultaría imposible evaluar los ítems tal y como se presentaban, los adecuamos a conceptos más cercanos a su actividad específica basándonos en contenidos integrados en actividades trabajadas en las sesiones de música, y que, en relación a las variables aquí presentadas son los que se reflejan en la Tabla 4, y aunque éstas no son habilidades 
que se hayan evaluado de forma específica, sí se han trabajado en el programa, por lo que pueden considerarse, a nuestro entender, beneficios “colaterales”.

En conclusión estamos razonablemente seguros de que la intervención realizada ha servido para corregir las dificultades rítmicas que los niños presentaban y que eran una de las causas de su problema, lo que ha ayudado, según la apreciación subjetiva de la propia logopeda, a que ocho de los nueve niños del grupo experimental hayan mejorado mucho en la producción de los fonemas afectados

Los resultados sugieren que el trabajo rítmico realizado a través de la música ha contribuido a rehabilitar las dislalias funcionales que presentaban, por tanto, y teniendo en cuenta todas las consideraciones hechas hasta ahora, podemos considerar que el programa ha cumplido con nuestras expectativas en relación con las variables presentadas.

En cuanto a la generalización de resultados creemos que, para obrar con rigor científico, sería necesario por un lado ampliar la muestra y por otro aumentar el número de sesiones de intervención si queremos afirmar con contundencia que los resultados se pueden generalizar; por ello el trabajo puede ser considerado como un estudio de caso.

\section{REFERENCIAS}

ACOSTA, V. M. y MORENO, A. Ma (2003): Dificultades del lenguaje, colaboración e inclusión educativa. Barcelona: Ars Médica.

BARRENA GONZÁLEZ, J. (2006): La dislalia. Marco conceptual, evaluación e intervención en el centro educativo. http://www.espaciologopédico.com

BUSTO BARCOS, M. C. (1998): Manual de logopedia escolar. Madrid: CEPE. CASTEJÓN FERNÁNDEZ, L. A. y ESPAÑA GANZARAIN, Y. (2004): La colaboración logopeda-maestro: hacia un modelo inclusivo en las dificultades del lenguaje. Revista de logopedia, foniatría y audiología, vol.24, $n^{\circ} 2$, 55-66.

GALÁN GALÁN, P. (2001): El logopeda como profesional del lenguaje y de la comunicación en los centros educativos. En Peñafiel, F. y Fernández, J. 
de D. (coords.) (2001): Cómo intervenir en logopedia escolar. Resolución de casos prácticos. Madrid: CCS.

GALLARDO RUIZ, J. R. y GALLEGO ORTEGA, J. L. (2003)(4 ${ }^{\mathrm{a}}$ edic.): Manual de logopedia escolar: un enfoque práctico. Málaga: Aljibe

GALLEGO ORTEGA, J. L. (2000): Dificultades de la articulación en el lenguaje oral. Málaga. Aljibe.

GARCÍA-VALDECASAS, M. (2001): Trastornos más usuales en el ámbito educativo. En Peñafiel, F. y Fernández, J. de D. (coords.) (2001): Cómo intervenir en logopedia escolar. Resolución de casos prácticos. Madrid: CCS.

GUBERINA, P.: Método verbotonal.

http://www.verbotonal.org/sistema-verbotonal/metodo-verbotonal.html HERRERA, L., HERNÁNDEZ-CANDELAS, M., LORENZO, O. y ROPP, C.(2014): Influencia del entrenamiento musical en el desarrollo cognitivo y lingüístico de niños de 3 a 4 años. Revista de psicodidáctica, $\quad$ 2014, $\quad$ 19(2), $\quad X X-\quad X X$. http://www.ehu.es/ojs/index.php/psicodidactica/article/view/9761/9763

JUAREZ SANCHEZ, A. y MONFORT, M. (1995): El niño que habla. Madrid: CEPE.

JUAREZ SANCHEZ, A. y MONFORT, M. (2001): Estimulación del lenguaje oral: un modelo interactivo para niños con necesidades educativas especiales. Madrid: Entha.

LÓPEZ GINÉS, M. J. et all. (1996): Exploración del lenguaje comprensivo y expresivo (ELCE). Madrid: CEPE.

MARTÍNEZ AGUDO, J. de D. et all. [eds..] (2002): Intervención en audición y lenguaje: casos prácticos. Madrid: EOS.

RAMÍREZ HURTADO, C. (2006): Música, lenguaje y educación: la comunicación humana a través de la música en el proceso educativo. Valencia: Tirant lo Blanch. 
RUIZ PALOMO, E. (2008): Programa de música para la rehabilitación de dislalias $y$ disfonías (intervención indirecta). http://redined.mecd.gob.es/xmlui/handle/11162/85087

SABBATELLA, P. L. (2001): Re-educación de la voz y el lenguaje en educación musical. Eufonía, $n^{\circ}$ 21, 98- 106.

VEIGA LIZ, M. P. (2004): Estimulación del lenguaje en Educación Infantil: guía práctica para docentes. Vigo: Ideaspropias.

VILASECA MOMPLET, R. (2002): La intervención logopédica en niños con dificultades en el área del lenguaje y la comunicación: un enfoque naturalista. Revista de logopedia, foniatría y audiología, vol. 22, $n^{\circ} 3$, 143- 150. 


\section{APÉNDICE A: INSTRUMENTO DE MEDIDA}

Apellidos... Nombre

Fecha de nacimiento

Edad

Curso

Tutor/a

\section{- PRUEBA DE RESPIRACIÓN}

Inspiración nasal-espiración nasal: ambas $\square$ una de las dos Inspiración nasal-espiración bucal: ambas $\square$ una de las dos Inspiración nasal-espiración bucal sostenida: ambas $\square$ una de las dos

- CAPACIDAD DE SOPLO

Soplo hinchando carrillos: Sí $\square$ No $\square$ Soplo sin hinchar carrillos: Sí $\square$ No $\square$ Soplo superior: Sí $\square$ No $\square$ Soplo inferior: Sí $\square$ No $\square$ Prueba del globo:

Realización: Sí $\square$ No $\square \quad$ Intentos:

Pérdidas de aire:

\section{- HABILIDAD BUCO-LINGUO-LABIAL}

Sacar-meter la lengua de la boca: Sí $\square$ No $\square$

Tocar la nariz con la punta de la lengua: Sí $\square$ No $\square$

Mover la lengua a izquierda-derecha fuera de la boca: Sí $\square$ No $\square$ Vibrar la punta de la lengua contra los dientes superiores: Sí $\square$ No $\square$ Mover la punta de la lengua por el paladar hacia atrás y adelante: Sí $\square$ No $\square$ Doblar la punta de la lengua dentro de la boca arriba-abajo: Sí $\square$ No $\square$ Apretar-aflojar los labios: Sí $\square$ No $\square$ Unir-separar rápidamente los labios: Sí $\square$ No $\square$ Imitar una moto: Sí $\square$ No $\square$ Morder el labio superior con los dientes inferiores: Sí $\square$ No $\square$ Morder el labio inferior con los dientes superiores: Sí $\square$ No $\square$

- PRUEBA DE ROSENTHAL O DE SUFICIENCIA NASAL Ambas fosas nasales: Sí $\square$ No $\square$ Intentos: Fosa nasal izquierda: Sí $\square$ No $\square$ Intentos: Fosa nasal derecha: Sí $\square$ No $\square \quad$ Intentos:

- ADECUACIÓN A UN RITMO EXTERNO

Adecuación a un ritmo lento: Sí $\square$ No $\square$ Adecuación a un ritmo normal: Sí $\square$ No $\square$ Adecuación a un ritmo rápido: Sí $\square$ No $\square$ 


\section{- REPETICIÓN DE ESTRUCTURAS RÍTMICAS}

$\begin{array}{lll}\text { Estructura } 1 & \ldots & \text { Sí } \square \text { No } \square \\ \text { Estructura } 2 & \ldots & \text { Sí } \square \text { No } \square \\ \text { Estructura } 3 & \ldots . . & \text { Sí } \square \text { No } \square \\ \text { Estructura } 4 & \ldots . & \text { Sí } \square \text { No } \square \\ \text { Estructura } 5 & \ldots . & \text { Sí } \square \text { No } \square \\ \text { Estructura } 6 & \ldots . & \text { Sí } \square \text { No } \square \\ \text { Estructura } 7 & \ldots . . . & \text { Sí } \square \text { No } \square\end{array}$

- DISCRIMINACIÓN AUDITIVA

- Discriminación de sonidos sin apoyo visual:

Prueba del bolígrafo y la caja: Sí $\square$ No $\square \quad$ Intentos:

Prueba de chinchetas y canicas: Sí $\square$ No $\square \quad$ Intentos:

- Discriminación de intensidad:

Prueba del bolígrafo y la caja: Sí $\square$ No $\square \quad$ Intentos:

Prueba de chinchetas y canicas: Sí $\square$ No $\square \quad$ Intentos:

- Discriminación de timbre:

Prueba del silbato y la flauta: Sí $\square$ No $\square \quad$ Intentos:

- Discriminación de tono:

Flauta, tono grave: Sí $\square$ No $\square \quad$ Intentos:

Flauta, tono agudo: Sí $\square$ No $\square \quad$ Intentos:

- Repetición de sonidos:

Prueba del reloj: Sí $\square$ No $\square \quad$ Intentos: 
EL TRABAJO RÍTMICO REALIZADO A TRAVÉS DE LA MÚSICA: UNA HERRAMIENTA PARA LA REHABILITACIÓN DE NIÑOS/AS CON DISLALIA FUNCIONAL

$\mathrm{M}^{\mathrm{a}}$ Esther Ruiz Palomo y Fernando Lara Ortega

Revista de Didácticas Específicas, nº 12, PP. 76-97

\section{APÉNDICE B}

Guía de observación

NOMBRE Y APELLIDOS:

\section{EDAD:}

\begin{tabular}{|c|c|c|c|c|c|c|c|c|c|c|c|c|c|c|c|}
\hline & 1 & 2 & 3 & 4 & 5 & 6 & 7 & 8 & 9 & 10 & 11 & 12 & 13 & 14 & 15 \\
\hline $\begin{array}{l}\text { Respiración } \\
\text { general }\end{array}$ & & & & & & & & & & & & & & & \\
\hline $\begin{array}{l}\text { Control } \\
\text { respiratorio }\end{array}$ & & & & & & & & & & & & & & & \\
\hline Emisión & & & & & & & & & & & & & & & \\
\hline Soplo & & & & & & & & & & & & & & & \\
\hline Articulación & & & & & & & & & & & & & & & \\
\hline Vocalización & & & & & & & & & & & & & & & \\
\hline $\begin{array}{l}\text { Reconoce } \\
\text { cualidades }\end{array}$ & & & & & & & & & & & & & & & \\
\hline Repite sonidos & & & & & & & & & & & & & & & \\
\hline $\begin{array}{l}\text { Movimiento } \\
\text { adecuado }\end{array}$ & & & & & & & & & & & & & & & \\
\hline $\begin{array}{l}\text { Palmea } \\
\text { palabras }\end{array}$ & & & & & & & & & & & & & & & \\
\hline $\begin{array}{l}\text { Palmea frases } \\
\text { (ritmo) }\end{array}$ & & & & & & & & & & & & & & & \\
\hline $\begin{array}{l}\text { Palmea pulsos/ } \\
\text { Acentos }\end{array}$ & & & & & & & & & & & & & & & \\
\hline $\begin{array}{l}\text { Repetición de } \\
\text { ritmos }\end{array}$ & & & & & & & & & & & & & & & \\
\hline
\end{tabular}

- : mal; r-: no del todo mal; r: regular; r+: casi bien; b: bien; b+: casi perfecto; mb: perfecto.

Observaciones:

Didácticas Específicas, ISNN: 1989-5240

www.didacticasespecificas.com 


\section{APÉNDICE C: TABLA PARA LA VALORACIÓN DE LA LOGOPEDA}

ALUMNO:

EDAD:

\begin{tabular}{|c|l|l|l|l|l|}
\hline & NADA & POCO & REGULAR & MUCHO & NOTABLEMENTE \\
\hline $\begin{array}{c}\text { Respiración } \\
\text { general }\end{array}$ & & & & & \\
\hline $\begin{array}{c}\text { Control } \\
\text { respiratorio }\end{array}$ & & & & & \\
\hline $\begin{array}{c}\text { Soplo } \\
\text { Emisión }\end{array}$ & & & & & \\
\hline $\begin{array}{c}\text { Articulación } \\
\text { Vocalización }\end{array}$ & & & & & \\
\hline $\begin{array}{c}\text { Ritmo y } \\
\text { acentuación } \\
\text { del habla }\end{array}$ & & & & & \\
\hline $\begin{array}{c}\text { Entonación } \\
\text { Expresividad }\end{array}$ & & & & & \\
\hline $\begin{array}{c}\text { Coordinación } \\
\text { dinámica } \\
\text { general }\end{array}$ & & & & & \\
\hline $\begin{array}{c}\text { Articulación } \\
\text { foctado/s }\end{array}$ & & & & & \\
\hline
\end{tabular}

OBSERVACIONES: 